\title{
Diagnostic Accuracy in Acute Venous Thromboembolism: Comparing D-Dimer, Thrombin Generation, Overall Hemostatic Potential, and Fibrin Monomers
}

\author{
${ }^{1}$ Department of Molecular Medicine and Surgery, Karolinska \\ Institutet, Stockholm, Sweden \\ ${ }^{2}$ Clinical Chemistry, Karolinska University Hospital, Stockholm, Sweden \\ ${ }^{3}$ Rheumatology Unit, Department of Medicine, Karolinska Institutet, \\ Stockholm, Sweden \\ ${ }^{4}$ Academic Specialist Center, Center for Rheumatology, Stockholm \\ Health Services, Stockholm, Sweden \\ ${ }^{5}$ Department of Medicine, Solna, Karolinska Institutet, Stockholm, \\ Sweden \\ ${ }^{6}$ Coagulation Unit, Division of Haematology, Karolinska University \\ Hospital, Stockholm, Sweden \\ ${ }^{7}$ Emergency Medicine Function, Karolinska University Hospital, \\ Stockholm, Sweden \\ 8 Division of Diagnostics and Specialist Medicine, Unit of Internal \\ Medicine, Medicine and Caring Sciences, Department of Health, \\ Linköping University, Linköping, Sweden
}

Maria Farm ${ }^{1,2}$ Aleksandra Antovic ${ }^{3,4}$ David E. Schmidt ${ }^{5,6}$ Niklas Bark $^{2}$ Nida Soutari ${ }^{1,2}$ Anwar J. Siddiqui ${ }^{7}$ Margareta Holmström ${ }^{8}$ Iva Pruner ${ }^{1}$ Jovan P. Antovic ${ }^{1,2}$

Address for correspondence Maria Farm, MD, PhD, Clinical Chemistry, L7:00, Karolinska University Hospital, Solna, Stockholm, SE-171 76, Sweden (e-mail: maria.farm@sll.se).

TH Open 2020;4:e178-e188.

\author{
Abstract \\ Keywords \\ - predictive value of \\ tests \\ - global hemostatic \\ assays \\ - clinical studies \\ - deep vein \\ thromboses \\ - pulmonary embolism
}

Introduction For acute venous thromboembolism (VTE), a biomarker with higher specificity than D-dimer would be of great clinical use. Thrombin generation and overall hemostatic potential (OHP) reflect the hemostatic balance by globally assessing multiple coagulation factors and inhibitors. These tests discriminate between healthy controls and patients with a prothrombotic tendency but have yet to be established as clinical biomarkers of VTE.

Objective This study compares endogenous thrombin potential (ETP) and OHP to Ddimer and fibrin monomers (FM) in outpatients with suspected VTE.

Methods A cross-sectional diagnostic study where 954 patients with suspected pulmonary embolism or deep venous thrombosis were recruited consecutively from the medical emergency department at Karolinska University Hospital. D-dimer, FM, OHP, and ETP were analyzed in a subpopulation of 60 patients with VTE and 98 matched controls without VTE. VTE was verified either by ultrasonography or computed tomography and clinical data were collected from medical records.

Results Compared with healthy controls, both VTE and non-VTE patients displayed prothrombotic profiles in OHP and ETP. D-dimer, FM, ETP area under the curve (AUC), and ETP $\mathrm{T}_{\text {lag }}$ were significantly different between patients with VTE and non-VTE. The largest receiver-operating characteristic AUCs for discrimination between VTE and non- received

February 5, 2020 accepted after revision June 9,2020
DOI https://doi.org/

10.1055/s-0040-1714210. ISSN 2512-9465. (c) 2020 Georg Thieme Verlag KG Stuttgart · New York
License terms

(c) (i) 
VTE, were found in D-dimer with 0.94, FM 0.77, and ETP AUC 0.65. No useful cutoff could be identified for the ETP or the OHP assay.

Conclusion Compared with D-dimer, neither ETP nor OHP were clinically viable biomarkers of acute venous thrombosis. The data indicated that a large portion of the emergency patients with suspected VTE were in a prothrombotic state.

\section{Introduction}

Venous thromboembolism (VTE) is the third most common cardiovascular disease and a cause of substantial morbidity and mortality worldwide. ${ }^{1}$ A definite diagnosis of VTE can generally not be reached clinically, so the quality of assays and imaging techniques are of utmost importance. While imaging is needed to verify VTE, the use of biomarkers in select cases can reduce time, cost, and iatrogenic complications in the diagnostic process. ${ }^{2,3}$ The only biomarker in common clinical use for diagnosis of VTE is D-dimer, the degradation product of polymerized and cross-linked fibrin. Fibrin monomers (FM) can possibly improve diagnosis if used together with D-dimer for the exclusion of VTE. ${ }^{4}$ The specificity of D-dimer is low at the chosen cutoff, with false positive results in up to $30 \%$ of tested patients with suspected VTE. $^{5}$ The low specificity is partially due to increased levels in patients with comorbidities. ${ }^{6}$ Increasing the effectiveness of the diagnostic process could potentially have major clinical and economical beneficial effects on the management of VTE.

Global hemostatic assays (GHAs) are a class of assays that examine the combined effect of pro- and anticoagulant processes in patient samples ex vivo. These assays monitor the whole coagulation process, either by using the clotting of whole blood or the generation of thrombin or fibrin in plasma as endpoints. While D-dimer reflects the in vivo activation of both hemostasis and fibrinolysis, the GHA illustrate the patients' current potential for these mechanisms. There is also the addition of a kinetic element, because the GHA continuously measure thrombin or fibrin generation and even fibrinolysis. Compared with D-dimer, global thrombin generation assays (TGAs) are also less influenced by comorbidities such as cancer, infectious disease, and cardiovascular disease. $^{7,8}$ A few studies have evaluated the use of TGA as a complement to D-dimer in the exclusion of $\mathrm{VTE}^{9,10}$ and indicated promising results of increased specificity paired with maintained sensitivity. TGA have showed prolonged time to peak $\left(\mathrm{T}_{\max }\right)$ and time to initiation of coagulation $\left(\mathrm{T}_{\text {lag }}\right)$ in acute VTE, possibly related to consumption of coagulation factors in the formation of the thrombus. ${ }^{8-12}$ In contrast, other studies have found an increased total thrombin generating capacity measured as the area under the curve (AUC), 8,9 which would not be the case after consumption of coagulation factors. Similarly, an increased thrombin generating capacity has been demonstrated in intermediate and serious thrombophilic phenotypes ${ }^{13}$ and has been associated with an increased risk of first VTE and unprovoked recurrent VTE, ${ }^{14,15}$ although there are conflicting results. ${ }^{16}$

In contrast to TGA, assays measuring the generation of fibrin, such as in the overall hemostatic potential (OHP) method, will also reflect patients' fibrinogen levels. ${ }^{17}$ The OHP can potentially be used for screening both hypo- and hypercoagulable conditions. ${ }^{18,19}$ It can also be used to characterize hypercoagulability or hypofibrinolysis in several prothrombotic conditions, such as antiphospholipid syndrome, ${ }^{20}$ after VTE, ${ }^{21,22}$ in acute coronary syndrome, ${ }^{23}$ and in acute stroke. ${ }^{24}$ Only a few studies have studied global fibrin generation in (semi-)acute venous thrombosis, these have demonstrated hypercoagulability and hypofibrinolysis compared with healthy controls. ${ }^{25,26}$

The Innovance endogenous thrombin potential (ETP) $)^{27}$ and the $\mathrm{OHP}^{28}$ assays are GHAs that have been optimized for analysis in routine coagulation laboratories. The OHP is a manual method that has been modified for routine laboratories and the ETP is an automated chromogenic TGA. Both assays feature simplified preanalytical handling and shortened analysis time, rendering them potentially useful in acute settings.

There is significant room for improvement of the biochemical diagnosis of VTE. Although some data point to the potential usefulness of the GHAs, a lack of standardization has hampered progress $^{29}$ and the clinical diagnostic value of ETP and OHP for diagnosis of VTE has not been formally assessed in a real-world clinical setting. This cross-sectional diagnostic study compares the diagnostic accuracy of OHP and ETP to D-dimer and FM for the assessment of suspect acute VTE in outpatients contacting the emergency department.

\section{Materials and Methods}

\section{Patients}

Samples were taken from the Karolinska Age Adjusted DDimer study (DFW-VTE). ${ }^{5}$ In short, 954 consecutive outpatients with clinically suspected pulmonary embolism (PE) or deep venous thrombosis (DVT) in the lower limb were prospectively recruited from the emergency department of Karolinska University Hospital in Huddinge, Sweden, between April 2014 and May 2015. Medical students were separately enrolled as healthy controls for post hoc analysis. The study was conducted in accordance with the Declaration of Helsinki and approved by the Regional Ethics Review Board in Stockholm (DNR 2013-2143-31-2). All participants provided written informed consent at enrolment.

\section{Samples}

Plasma samples were collected in $0.109 \mathrm{~mol} / \mathrm{L}$ (3.2\%) sodiumcitrate plastic vacutainer tubes (Becton Dickinson, New Jersey, United States) by direct venipuncture, without addition of corn trypsin inhibitor. ${ }^{29}$ Samples were collected at the emergency unit before initiation of any anticoagulant therapy. After centrifugation at $3,000 \times g$ for 10 minutes, samples were 
analyzed for D-dimer, FM, and prothrombin time (PT) (international normalized ratio [INR]) and then aliquoted and frozen at $-70^{\circ} \mathrm{C}$ within 1 hour. Samples were thawed in a $37^{\circ} \mathrm{C}$ water bath for analysis of ETP, OHP, fibrinogen, antithrombin, and Creactive protein (CRP). Because sample collection was performed in a routine clinical chemistry laboratory where storage of research samples could not be the main priority, only a subset of samples from the DFW-VTE study ${ }^{5}$ could be saved for analysis by FM and stored.

In this study, we included all samples with VTE and randomly selected age- and sex-matched samples without VTE (-Fig. 1). All included samples that were available (that had been stored) were analyzed by ETP and OHP ( $n=174 ; 62$ VTE and 112 without VTE). After exclusions 158 samples remained, as follows (-Fig. 1). Seven non-VTE samples were excluded because of low technical quality due to hemolysis, lipemia, or clotting. Nine patients were excluded due to anticoagulant treatment, which was initiated before arrival to the emergency department. In four samples, ETP was not analyzed due to insufficient sample volume (one VTE, three non-VTE).

Healthy controls were also analyzed for OHP and fibrinogen $(n=42)$ and ETP $(n=37)$ to further characterize the hypercoagulable state of the study subjects.

\section{Clinical Data}

Clinical data were collected from medical records by a resident MD blinded for the assay results. VTE had been verified radiologically by computed tomography or ultrasonography, as appropriate. VTE had been excluded radiologically $(n=49)$ and was otherwise excluded by a 3-month follow-up of medical records $(n=49)$. Radiology was accredited according to ISO/IEC 17025 by the Swedish Board for
Accreditation and Conformity Assessment and was performed on the day of sampling in 83 patients, the following day in 15 patients, 3 days after sampling in 3 patients and after 7 days in 2 patients. Isolated thrombophlebitis was classified as negative for VTE and was present in nine cases. Patient characteristics are described in - Table 1. The eight patients with cancer suffered from prostate cancer $(n=2)$, brain tumors $(n=2)$, malignant melanoma $(n=1)$, ovarian cancer $(n=1)$, hairy cell leukemia $(n=1)$, and liver metastasized cancer of unknown primary tumor $(n=1)$. Recent trauma or surgery $(n=7)$ was defined as an incident occurring less than 1 month before sampling. Prior thrombophilia testing in tested patients $(n=17)$, had consisted of lupus anticoagulant, antithrombin, protein $S$, protein $C$, and the genetic variants Factor V Leiden and Factor II G20210A.

\section{Assays}

Assays were performed blinded to clinical data and results of any other assay. Thrombin generation was analyzed using the automated Innovance ETP assay ${ }^{27,30}$ on the BCS XP System. The instrument, reagent, and calibrator were provided by Siemens Healthcare Diagnostics (Erlangen, Germany). The ETP was performed in platelet-poor plasma using B-settings, the proprietary recommended settings for patients with suspected hypercoagulability, activated by tissue factor (TF) in high concentration (300 PM). The reagent contains a nondefined fibrin aggregation inhibitor and a slow reacting thrombin chromophore substrate $(\mathrm{H}-\beta-$ Ala-Gly-Arg-pNA). The parameters are the area under the thrombin generation curve (ETP AUC), which corresponds to the total generation of thrombin, the peak thrombin concentration (ETP $C_{\max }$ ), the time to initiation of thrombin

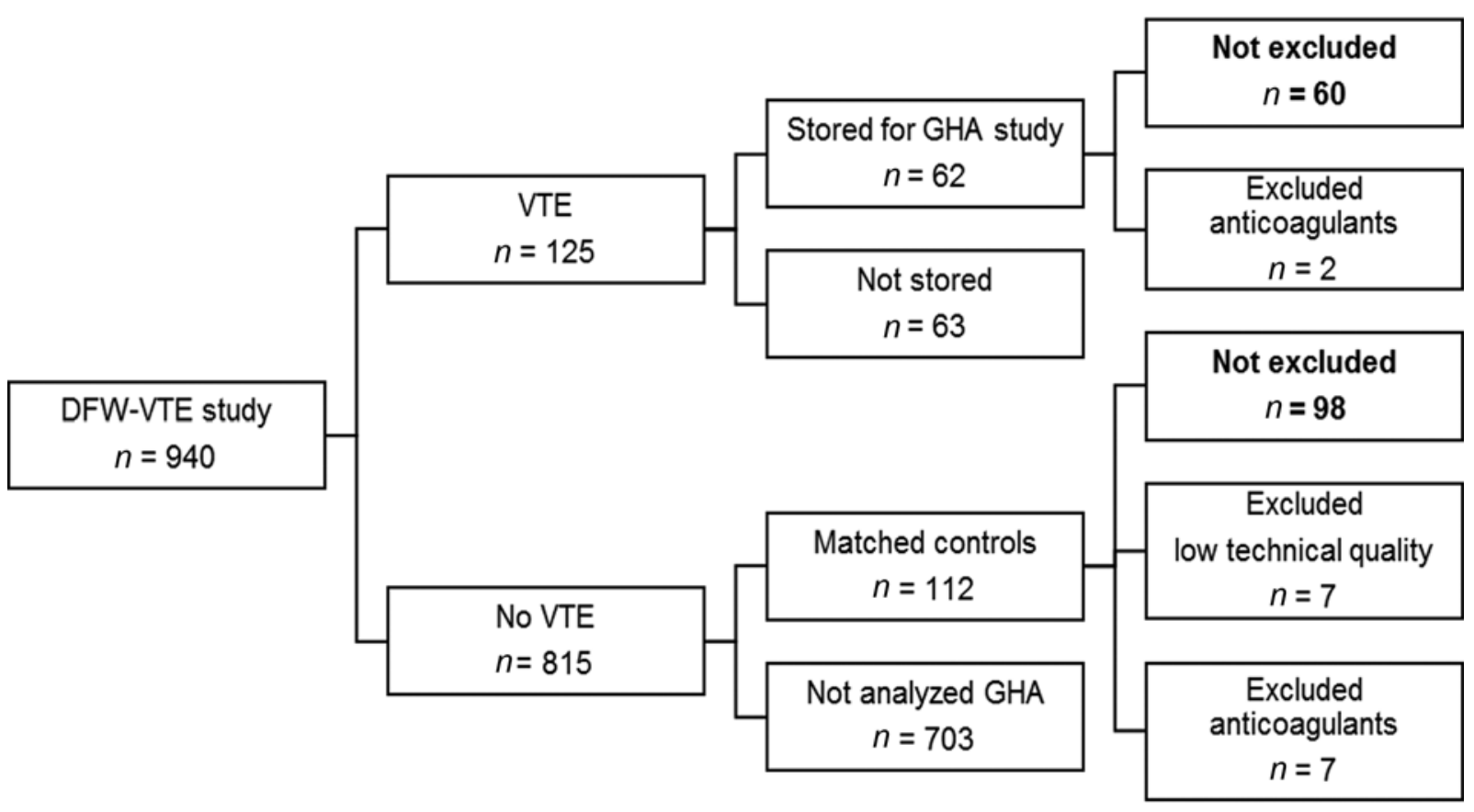

Fig. 1 Flowchart of study samples. Matched controls were selected for all patients with venous thromboembolism (VTE). Only a subset of the 940 samples could be stored in the acute clinical chemistry laboratory. All included samples $(n=174)$ were analyzed by overall hemostatic potential (OHP) and endogenous thrombin potential (ETP). Nine patients were excluded due to prior anticoagulant treatment and seven due to low technical quality. Primary analysis in this study was performed on 60 samples with VTE and 98 without VTE. 
Table 1 Patient characteristics for patients with and without VTE

\begin{tabular}{|c|c|c|c|c|}
\hline & & No VTE $(n=98)$ & VTE $(n=60)$ & $p$-Value \\
\hline Age $[y]$ & $\begin{array}{l}\text { Mean }(95 \% \mathrm{Cl}) \text {, } \\
\text { range }\end{array}$ & $\begin{array}{l}61(57-64) \\
24-91\end{array}$ & $\begin{array}{l}62(58-66) \\
20-96\end{array}$ & $0.668^{\mathrm{a}}$ \\
\hline PT (INR) $[<1.2]$ & $\begin{array}{l}\text { Median (IQR), } \\
\text { range }(n)\end{array}$ & $\begin{array}{l}1.0(1.00-1.10) \\
0.8-1.5(89)\end{array}$ & $\begin{array}{l}1.0(1.0-1.10) \\
0.98-1.10(57)\end{array}$ & $0.419^{b}$ \\
\hline Antithrombin, [0.8-1.2 kIU/L] & $\begin{array}{l}\text { Median (IQR), } \\
\text { range }(n)\end{array}$ & $\begin{array}{l}1.0(0.9-1.1) \\
0.6-1.5(82)\end{array}$ & $\begin{array}{l}1.0(0.9-1.1) \\
0.6-1.4(46)\end{array}$ & $0.194^{b}$ \\
\hline Fibrinogen $[2.0-4.2 \mathrm{~g} / \mathrm{L}]$ & $\begin{array}{l}\text { Median (IQR), } \\
\text { range }(n)\end{array}$ & $\begin{array}{l}3.5(3.0-4.2) \\
1.0-6.0(84)\end{array}$ & $\begin{array}{l}4.0(3.1-5.3) \\
1.2-7.5(46)\end{array}$ & $0.057^{b}$ \\
\hline $\mathrm{CRP}[<3 \mathrm{mg} / \mathrm{L}]$ & $\begin{array}{l}\text { Median (IQR), } \\
\text { range }(n)\end{array}$ & $\begin{array}{l}4(1-11) \\
1-104(98)\end{array}$ & $\begin{array}{l}11(4-44) \\
1-295(59)\end{array}$ & $<0.001^{b}$ \\
\hline Female & Proportion $(n)$ & $0.48(47)$ & $0.42(25)$ & \multirow[t]{2}{*}{$0.441^{c}$} \\
\hline Male & Proportion $(n)$ & $0.52(51)$ & $0.58(35)$ & \\
\hline Previous VTE & Proportion $(n)$ & $0.11(11)$ & $0.38(23)$ & $<0.001^{c}$ \\
\hline Positive D-dimer & Proportion (n) & $0.34(33)$ & $0.97(58)$ & $<0.001^{c}$ \\
\hline Trauma or surgery & Proportion $(n)$ & $0.01(1)$ & $0.10(6)$ & $0.012^{d}$ \\
\hline Cancer & Proportion $(n)$ & $0.08(8)$ & $0.07(4)$ & $1.000^{\mathrm{d}}$ \\
\hline Liver disease & Proportion $(n)$ & $0.07(7)$ & $0.00(0)$ & $0.045^{d}$ \\
\hline Pregnant & Proportion $(n)$ & $0.03(3)$ & $0.02(1)$ & $1.000^{\mathrm{d}}$ \\
\hline Platelet inhibitors & Proportion $(n)$ & $0.39(38)$ & $0.22(13)$ & $0.026^{\mathrm{C}}$ \\
\hline $\mathrm{PHC}$ & Proportion $(n)$ & $0.02(2)$ & $0.05(3)$ & $0.369^{d}$ \\
\hline Thrombophilia & Counts & 2 of 10 tested & 15 of 30 tested & \\
\hline
\end{tabular}

Abbreviations: $\mathrm{Cl}$, confidence interval; CRP, C-reactive protein; INR, international normalized ratio; IQR, interquartile range; PHC, prothrombotic hormonal contraceptives; PT, prothrombin time; VTE, venous thromboembolism.

Notes: No significance test was performed on the thrombophilia variable, because the patients without VTE had not been tested to a comparable extent. Boldface values signify $p$-values for significant differences.

andividual variables $t$-test.

${ }^{\mathrm{b}}$ Mann-Whitney $U$ test.

'Pearson's chi-square test.

${ }^{\mathrm{d}}$ Fischer's exact test.

generation (ETP $\mathrm{T}_{\text {lag }}$ ), and the time to peak thrombin generation (ETP $\mathrm{T}_{\mathrm{max}}$ ). Results were normalized against pooled normal plasma, giving results in \%. Intra-assay and interassay variation coefficient (CV\%) for the ETP AUC were 3.3 and $2.7 \%$, respectively.

OHP was performed in 96-well plates using the method modified for routine laboratories, as previously described. ${ }^{31}$ Two curves (with and without fibrinolysis initiated by tissue plasminogen activator) were used to calculate the area under the fibrin generation curve (overall coagulation potential, $\mathrm{OCP}$ ), the AUC in the well of fibrin generation plus fibrinolysis (OHP), and the decrease in fibrinogen concentration by fibrinolysis as a proportion of the OCP (overall fibrinolytic potential, OFP). Intra-assay and interassay $\mathrm{CV} \%$ for the OHP were 9.3 and $12.3 \%$, respectively.

D-dimer, FM, and PT (INR) were analyzed immediately on the Sysmex CS2100i instrument (Siemens). D-dimer was analyzed using the Tina-quant D-dimer (Roche Diagnostics, Basel, Switzerland). ${ }^{32}$ FM was analyzed using STA-Liatest FM (Diagnostica Stago, Asnières-sur-Seine, France). ${ }^{33}$ Both assays are rapid particle-enhanced immunoturbidimetric assays. PT (INR) was analyzed by MRX Owrens PT (Medirox, Nyköping, Sweden). ${ }^{34}$
Fibrinogen and antithrombin were analyzed on the Sysmex CS5100 instrument (Siemens) in all samples with remaining plasma after analysis of ETP and OHP. Fibrinogen was analyzed using the Dade thrombin reagent (Siemens) which is a modified Clauss assay. ${ }^{35}$ Antithrombin was analyzed with enzymatic the FII-based Berichrom Antithrombin III (Siemens) assay. ${ }^{36}$ CRP was analyzed with the immunoturbidimetric CRPL3, C-Reactive Protein Gen. 3 assay on the Cobas 6000 instrument (Roche Diagnostics). The exact number of samples that were available for each assay is given in -Tables $\mathbf{1}$ and $\mathbf{2}$.

\section{Statistical Methods}

Descriptive statistics are presented as a range, mean (with $95 \%$ confidence interval $[\mathrm{CI}]$ or \pm standard error of the mean), median, and interquartile range, as appropriate. Groups were compared using the independent samples $t$-test or by the nonparametric Mann-Whitney $U$ test. Multiple groups were compared by a Kruskal-Wallis rank-sum test. Proportions were tested by Pearson's chi-square or Fischer's exact test. Pretest probabilities were calculated to assure diagnostic comparability between the assays. To summarize the overall discriminatory value of the assays, receiver-operating characteristic (ROC) AUCs (ROC AUCs) ${ }^{37}$ were used. The 
e182 Diagnostic Accuracy of DDI, ETP, OHP, and FM Farm et al.

Table 2 Assay results and significant differences between patients with and without VTE

\begin{tabular}{|c|c|c|c|c|c|}
\hline Parameter & No VTE & VTE & $p$-Value & $\begin{array}{l}\text { Reference } \\
\text { interval }^{c}\end{array}$ & $\begin{array}{l}\text { Healthy controls, } \\
\text { central } 95 \\
\text { percentile }\end{array}$ \\
\hline OHP, mean ( \pm SEM) & $15.9(15.3-16.5)$ & $16.2(15.3-17.1)$ & $0.811^{\mathrm{a}}$ & $6.5-13.6$ & $6.3-16.2$ \\
\hline OCP, mean $( \pm$ SEM) & $25.0(24.3-25.7)$ & $26.5(38.5-41.3)$ & $0.166^{\mathrm{a}}$ & $12.7-24.0$ & $14.5-27.8$ \\
\hline OFP, mean $( \pm$ SEM) & $37(36-39)$ & $40(38-41)$ & $0.166^{\mathrm{a}}$ & $31-57$ & $38-58$ \\
\hline ETP AUC, mean ( \pm SEM) & $99(97-100)$ & 107 (106-109) & $0.001^{\mathrm{a}}$ & $87-128$ & $74-112$ \\
\hline ETP $C_{\max }$, median (IQR) & $111(99-122)$ & $113(103-127)$ & $0.136^{\mathrm{b}}$ & $82-119$ & $78-109$ \\
\hline ETP $t_{\text {lag }}$, median (IQR) & $21.2(19.6-22.6)$ & $22.0(20.3-23.9)$ & $0.031^{b}$ & - & $22-30$ \\
\hline ETP $t_{\text {max }}$, mean $( \pm S E M)$ & $56.4(55.6-57.2)$ & $54.4(53.5-55.4)$ & $0.126^{\mathrm{a}}$ & - & $59-85$ \\
\hline D-dimer, median (IQR) & $0.38(0.22-0.69)$ & $2.93(1.55-12.27)$ & $<0.001^{\mathrm{b}}$ & $<0.5$ & - \\
\hline $\begin{array}{l}\text { Fibrin monomers, } \\
\text { median (IQR) }\end{array}$ & $3(1-4)$ & $9(3-117)$ & $<0.001^{b}$ & $<6$ & - \\
\hline
\end{tabular}

Abbreviations: AUC, area under the curve; ETP, endogenous thrombin potential; IQR, interquartile range; OHP, overall hemostatic potential; SEM, standard error of the mean; VTE, venous thromboembolism.

Notes: With VTE, $n=98$ (D-dimer, OHP, OCP, OFP) and $n=95$ (fibrin monomers, ETP AUC, ETP $C_{\max }$, ETP $_{\text {lag }}$, ETP $t_{\text {max }}$ ). Without VTE, $n=60$ (D-dimer, OHP, OCP, OFP) and $n=59$ (fibrin monomers, ETP AUC, ETP $C_{\text {max }}$, ETP $t_{\text {lag }}$, ETP $t_{\text {max }}$ ).

Boldface values signify $p$-values for significant differences.

andependent samples $t$-test.

${ }^{\mathrm{b}}$ Mann-Whitney $U$ test.

${ }^{\mathrm{C}}$ Reference intervals OHP ${ }^{31}$ and ETP. ${ }^{27}$

diagnostic accuracy of the parameters of the OHP and ETP assays and FM were compared by sensitivity and specificity at the level where the sensitivity was equal to the sensitivity of D-dimer in the cohort. The association of ETP AUC with VTE status was examined by binomial logistic regression. ETP and OHP results were visually compared in clinical subgroups. Odds ratio (OR) for VTE was calculated for OCP, before and after adjusting for fibrinogen levels. Statistical analysis was performed using SPSS 23, MS Excel, and R version 3.6.0. $p$-Values $<0.05$ were considered significant.

\section{Results}

\section{Patient Characteristics}

The study cohort included 60 patients with VTE and 98 randomly selected patients with non-VTE (-Fig. 1). VTE was localized as PE in 16 patients, proximal DVT in 25, and distal DVT, isolated below popliteal level in 19 patients. Thrombophlebitis was found in 10 patients and was classified as negative for VTE. Patient characteristics are summarized in - Table 1. Patients with VTE had significantly higher CRP results (median $11 \mathrm{vs.} 4 \mathrm{mg} / \mathrm{L}, p<0.001$ ), frequency of positive D-dimer $(0.97 \mathrm{vs.} 0.34, p<0.001)$, and prevalence of previous VTE ( 0.38 vs. $0.11, p<0.001)$ and of recent trauma or surgery ( 0.10 vs. $0.01, p=0.012)$. They also had a lower prevalence of treatment with platelet inhibitors $(0.22 \mathrm{vs}$. $0.39, p=0.026)$ and of liver disease ( 0.00 vs. $0.07, p=0.045$ ).

\section{Assessment of ETP and OHP in Patients with Suspected VTE}

The pretest probability was 0.38 for all evaluated assays. Box and whisker plots of assay results in patients with VTE, without VTE, and healthy controls are presented in - Fig. 2. All OHP and ETP parameters, as well as fibrinogen, demonstrated pro- thrombotic profiles in patients with clinically suspected VTE with significant differences compared with healthy controls (Kruskal-Wallis rank-sum test: $p<0.005$ ).

Assay differences between patients with and without VTE are presented in - Table $\mathbf{2}$. Significant differences were found in D-dimer and FM $(p<0.001)$, ETP $\mathrm{T}_{\operatorname{lag}}(p=0.031)$, and for ETP AUC $(p=0.001)$. ETP AUC was associated with VTE with an OR of 1.04 (95\% CI, 1.02-1.07), given a one-unit increase in ETP AUC. OCP was not associated with VTE in univariate analysis, OR 1.03 (95\% CI, $0.99-1.08 ; p=0.17$ ), or after adjusting for fibrinogen levels, OR 0.94 (95\% CI, 0.85-1.05; $p=0.27$ ). Reference intervals for ETP and $\mathrm{OHP}^{27,31}$ and the central 95 percentiles of healthy controls are also presented in - Table 2 .

Outliers (not excluded, - Fig. 2) consisted of a patient with large central bilateral PE with high OHP and OCP, and a high outlier in ETP AUC with a recent proximal humerus fracture. ETP $C_{\max }$ had a low outlier with hemoglobin 35 and a high outlier with thrombophilia and DVT and ETP $\mathrm{T}_{\text {lag }}$ had several outliers. ETP $\mathrm{T}_{\max }$ had two nonpathological outliers, while most other patients had shortened times to peak.

To investigate potential skewing by third variables, we also assessed the relationship between ETP AUC and OHP levels with age, gender, and previous VTE ( - Fig. 3 ). In this analysis, patients with pregnancy, cancer, liver pathology, and recent trauma or surgery were excluded (remainder; $n=125$ ). ETP AUC showed a decrease with increasing age, whereas OHP did not correlate with age. ETP AUC was also increased in females, where the difference between patients with and without VTE was more pronounced than in the group as a whole. OHP was less increased in patients with previous VTE than without, regardless of current VTE status. Treatment with platelet inhibitors was significantly more common in controls than in patients but affected neither ETP AUC nor OHP. Due to low numbers, it was not possible to 

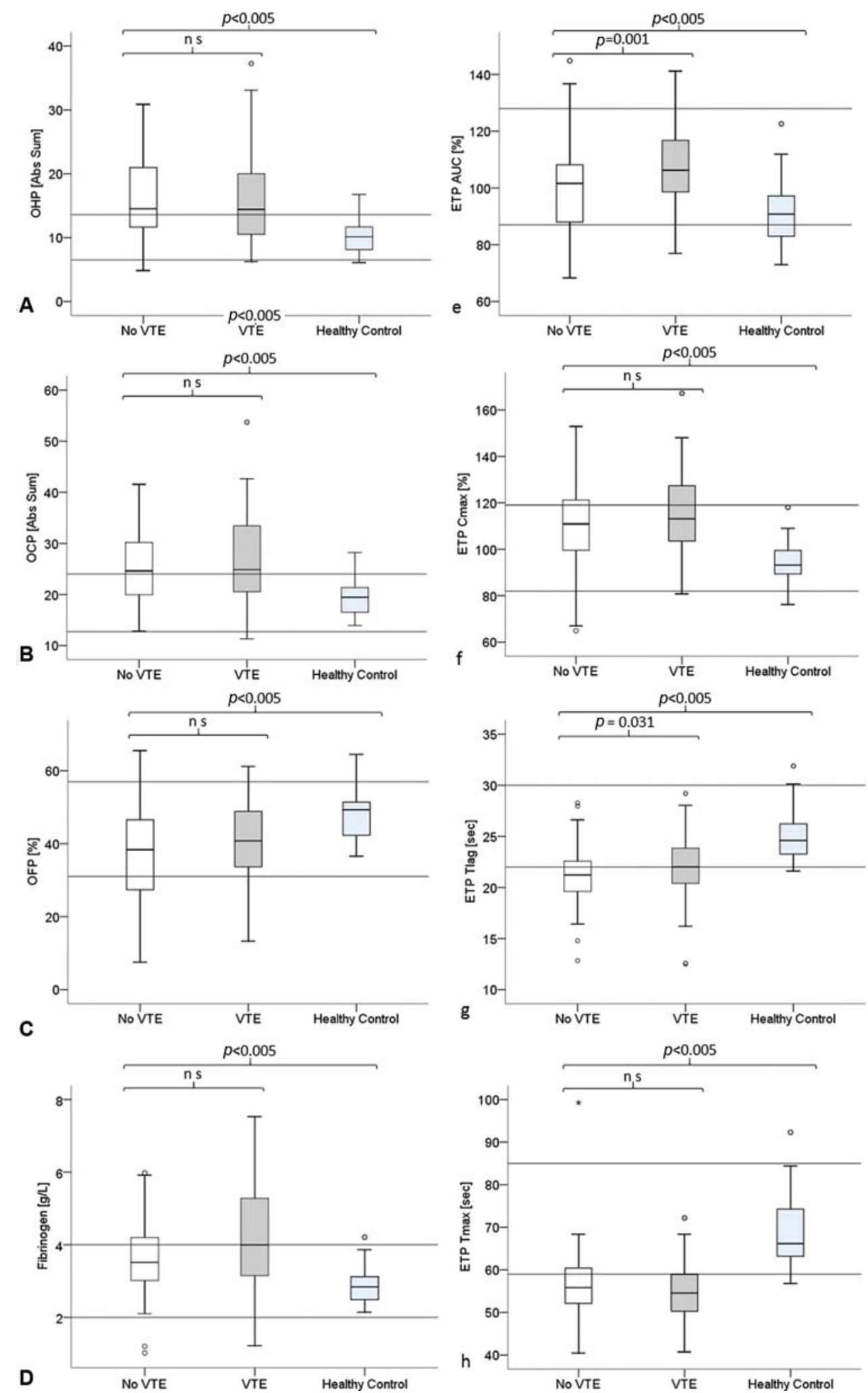

Fig. 2 (A) Overall hemostatic potential (OHP), (B) OCP, (C) OFP, (D) fibrinogen, (E) endogenous thrombin potential area under the curve (ETP AUC), (F) ETP $\mathrm{C}_{\max },(\mathrm{G}) \mathrm{ETP} \mathrm{T}_{\text {lag, }}$ and (H) ETP $\mathrm{T}_{\max }$. Difference in global hemostatic parameters between patients with no venous thromboembolism (VTE) [white], VTE [gray], and healthy controls [light gray]. Box and whisker plots displaying medians [mid-line], interquartile range (IQR) [box], $1.5 \times$ IQR [whisker], outliers $>1.5 \times$ IQR [ring], and extreme outliers $>3 \times$ IQR [asterisk].

assess the occurrence of any trends in the relationship between ETP AUC or OHP and recent trauma/surgery, cancer, or liver pathology $(n=158)$ in relation to VTE status (-Supplementary Fig. S1).
Finally, considering a full diagnostic model of VTE that included trauma, liver disease, recent pregnancy, thrombophilia, cancer state, gender, and D-dimer, the effect of ETP AUC was not significant (data not presented). 

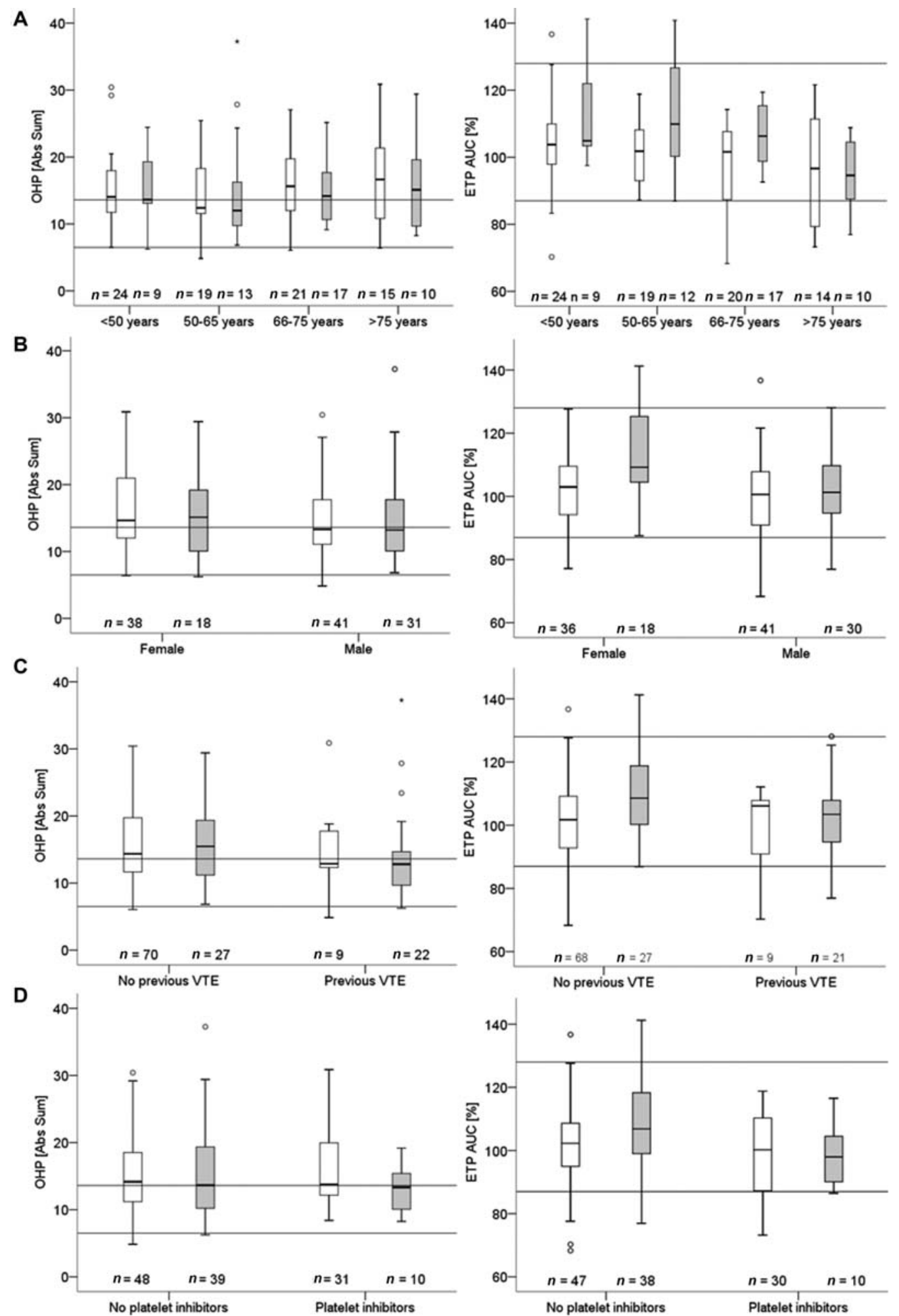

Fig. 3 (A) Age, (B) gender, (C) previous venous thromboembolism (VTE), and (D) platelet inhibitors. Box and whisker plots of endogenous thrombin potential area under the curve (ETP AUC) and overall hemostatic potential (OHP) related to age, gender, and previous VTE (samples in analysis $=125)$. No VTE [white], VTE [gray], medians [mid-line], interquartile range (IQR) [box], $1.5 \times \operatorname{IQR}$ [whisker], outliers $>1.5 \times \operatorname{IQR}$ [ring], and extreme outliers $>3 \times$ IQR [asterisk].

Assessment of Diagnostic Accuracy of ETP and OHP for VTE

For discrimination of radiology-confirmed VTE among patients with suspected VTE, all parameters of OHP and ETP had ROC AUCs $\leq 0.65$ (-Table 3; - Fig. 4). The ROC
AUC of D-dimer was 0.94 and FM 0.76, while the largest AUC of the global hemostatic parameters was ETPAUC at 0.65. The specificities ranged from 0.00 to 0.20 at the respective cutoffs where the sensitivities of each parameter was 0.97 (in accordance with the Clinical and Laboratory Standards 
Table 3 Diagnostic accuracy of the evaluated assays presented as areas under the ROC curve and as sensitivity and specificity at a cutoff with sensitivity equal to D-dimer and the CLSI requirements for assays to exclude venous thrombosis

\begin{tabular}{|l|l|l|l|l|}
\hline Parameter & ROC AUC $(95 \% \mathrm{Cl})$ & Cutoff & Sensitivity & Specificity \\
\hline OHP [Abs Sum] & $0.50(0.40-0.60)$ & $<7$ & 0.97 & 0.04 \\
\hline OCP [Abs Sum] & $0.55(0.45-0.65)$ & $<12$ & 0.97 & 0.00 \\
\hline OFP [\%] & $0.56(0.47-0.65)$ & $<17$ & 0.97 & 0.09 \\
\hline ETP AUC [\%] & $0.65(0.56-0.74)$ & $<85$ & 0.97 & 0.20 \\
\hline ETP $C_{\max }[\%]$ & $0.57(0.48-0.67)$ & $<90$ & 0.97 & 0.13 \\
\hline ETP T lag $[\mathrm{s}]$ & $0.60(0.51-0.70)$ & $<15$ & 0.97 & 0.02 \\
\hline ETP T $\max [\mathrm{s}]$ & $0.43(0.33-0.52)$ & $<42$ & 0.97 & 0.01 \\
\hline D-dimer [mg/L FEU] & $0.94(0.90-0.97)$ & $<0.5$ & 0.97 & 0.66 \\
\hline Fibrin monomers [mg/L] & $0.76(0.68-0.85)$ & $<0.9$ & 0.97 & 0.20 \\
& & $<6.0$ & 0.57 & 0.84 \\
\hline
\end{tabular}

Abbreviations: Abs Sum, sum of the absorbances; Cl, confidence interval; CLSI, Clinical and Laboratory Standards Institute; ETP, endogenous thrombin potential; FM, fibrin monomers; FEU, fibrin equivalent units; OHP, overall hemostatic potential; ROC AUC, area under the receiveroperating characteristic curve.

Note: For FM, sensitivity and specificity at the current proprietary cutoff $(<6.0 \mathrm{mg} / \mathrm{L})$ is also presented.

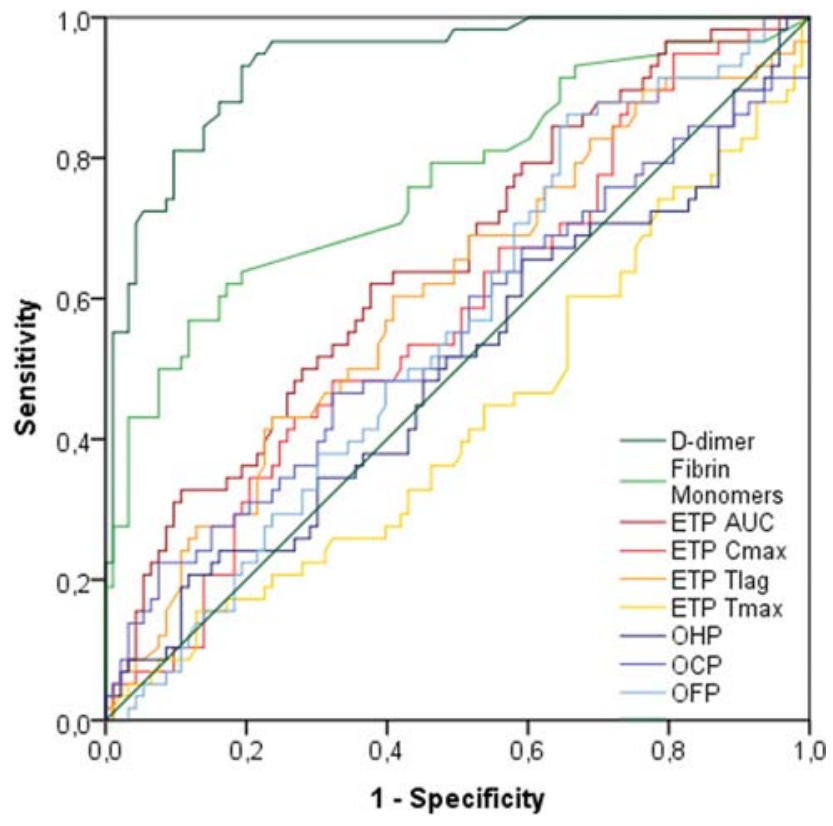

Fig. 4 Receiver-operating characteristic (ROC) curves for global hemostatic assays, D-dimer, and fibrin monomers. D-dimer and fibrin monomers are plotted in green, overall hemostatic potential (OHP) parameters in blue, and endogenous thrombin potential (ETP) parameters in red-orange. ETP $\mathrm{T}_{\max }$ (light orange) is negative because it is the only parameter where a smaller test result indicates a more "positive" test.

Institute [CLSI] recommendations ${ }^{38}$ and equal to the sensitivity of D-dimer in the cohort).

Similarly, an exploration of other cutoffs to minimize the distance to the upper left corner of the ROC (the Euclidean distance between the ROC curve and the point where sensitivity and specificity are both 1.0) corresponded to poor tradeoffs of sensitivity and specificity, with sensitivities and specificities in the region of 0.40 to 0.70 (-Table 3 ).

Distal DVT was the main pathology in $32 \%$ of the patients with VTE, and low thrombotic load may decrease an evaluated diagnostic sensitivity. When we excluded these patients and restricted analyses to only the cases with proximal DVT and (segmental) PE, the ROC AUC increased by only -0.02 to 0.05 (data not presented). When we excluded the nine patients with thrombophlebitis from the patient controls without VTE, ROC AUC increased by 0.01 for OCP, OFP, and ETP $\mathrm{T}_{\text {lag. }}$. Differences between patients with and without VTE did not become significant by these restrictions.

\section{Additive Diagnostic Value after Initial D-Dimer Testing} In the subgroup of patients with positive D-dimer, we could not observe any increase in sensitivity/specificity or ROC AUC of ETP or OHP (data not presented). Given the association of ETP AUC with VTE, we also assessed the potential value of ETP AUC after D-dimer testing. Only two patients with VTE had a negative D-dimer; both had an ETP AUC $>100 \%$. Among patients with a positive D-dimer, $17 / 37(46 \%)$ patients with ETPAUC $<100 \%$ had a VTE; compared with 40/53 (75\%) with an ETP AUC $\geq 100 \%$. Although the ETP AUC was associated with VTE in D-dimer-positive patients, it insufficiently discriminated VTE from non-VTE (data not presented).

\section{Discussion}

We performed a cross-sectional single-center diagnostic study to assess the clinical value of two plasma-based GHAs in patients with suspected acute VTE (DVT or PE), compared with D-dimer and FM. We were able to confirm the previously described increase of ETP AUC ${ }^{8,9}$ and ETP $\mathrm{T}_{\text {lag }}{ }^{8-12}$ in acute VTE. However, the significant differences were appreciably smaller than for D-dimer and insufficient to discriminate between patients with and without VTE. These results are in line with recent clinical evaluations of other TGA for acute VTE. ${ }^{10}$ Our results indicate that neither the ETP nor the OHP assay would be clinically useful additions as biomarkers for the diagnosis of acute VTE in the emergency department.

A biomarker to replace or complement D-dimer would need to exhibit a robust specificity at a cutoff chosen to have 
at least the same sensitivity as D-dimer. The CLSI recommendations for D-dimer assays for the exclusion of VTE, state that sensitivity at the chosen cutoff must be $\geq 0.97(\geq 0.90$, lower limit 95\% CI). ${ }^{38}$ Application of this criterion to the GHA demonstrated that none of the ETP and OHP parameters can achieve such sensitivity while maintaining a useful specificity.

Interestingly, patients with suspected VTE showed significantly increased fibrinogen levels compared with the healthy controls, regardless of final VTE status. The OHP and OCP were also increased and OFP was decreased. These results indicate that a large portion of the emergency patients were in a prothrombotic state, which could be explained in part by increased fibrinogen levels. This could be considered a factor that makes the OHP unsuitable for exclusion of VTE in emergency department. The prothrombotic tendency was not observed to the same extent in the ETP assay, which is analyzed in defibrinated samples, although ETP $\mathrm{T}_{\max }$ and ETP $\mathrm{T}_{\text {lag }}$ were shortened in both groups and $\mathrm{ETP}_{\max }$ was increased. ETPAUC seemed to be least affected, in accordance with studies that suggest it is less influenced by some comorbidities than Ddimer. ${ }^{7,8}$ However, ETP AUC was not superior to D-dimer for discrimination of VTE in this real-life cohort with a relatively high comorbidity burden. The use of TGAs in acute settings may indeed be prone to acute phase effects which have not been extensively evaluated yet. One such issue that impacts the accuracy of the TGAs, is the existence of $\alpha$-2-macroglobulin $(\alpha 2 \mathrm{M})$-thrombin complexes in plasma. The Innovance ETP assay attempts to correct for the presence of a fixed amount of $\alpha 2 \mathrm{M}$-trombin via mathematical calculations, but levels of $\alpha 2 \mathrm{M}$ can vary greatly by age and by conditions such as hepatitis $C$, pancreatitis, or acute ischemic heart disease, ${ }^{39}$ which introduces an interindividual variation in the physiological relevance of TGAs. It is possible that several of the patients in this study had abnormal levels of $\alpha 2 \mathrm{M}$, which may have influenced the results of the ETP assay.

To evaluate the possible use of Innovance ETP as a secondtier analysis for VTE, we analyzed the diagnostic accuracy in the subgroup of patients with positive D-dimer and observed no increase in sensitivity/specificity or ROC AUC. Haas et $\mathrm{al}^{9}$ suggested that for patients $>75$ years with positive $D$-dimer and low Wells score, the Innovance ETP $\mathrm{T}_{\text {Lag }}$ could be used as a second-tier analysis in the emergency department. This was based on a group of 30 patients, where ROC AUC was 0.96 using a cutoff of 23 seconds, sensitivity 1.00 , and specificity 0.96 . In our cohort, 29 patients were above 75 years with a positive D-dimer. In this group, the ROC AUC for ETP $\mathrm{T}_{\text {Lag }}$ was 0.49 , if the same cutoff of 23 seconds was applied. The sensitivity was only 0.46 paired with a specificity of 0.75 (data not presented). In conclusion, our findings contradict the use of ETP $\mathrm{T}_{\text {Lag }}$ as a second-tier analysis in patients with unselected clinical probability of VTE.

\section{Limitations}

Only a limited set of matched control samples could be analyzed due to logistical reasons previously explained. However, we minimized the risk of selection bias by choosing pairs at random after matching for age and sex.
Given our study size, it was not possible to stratify patients with PE and proximal or distal DVT. Distal DVT was the main pathology in $32 \%$ of the patients with VTE, that is, cases where surveillance is often recommended over anticoagulant treatment, though the vast majority is treated with anticoagulants. ${ }^{40}$ However, our results did not change when we excluded patients with distal DVT from the analysis.

The results of the GHA may be affected by the possible presence of thrombophilia in some subjects, although this does not affect the conclusion of the study. It would have been very interesting to acquire information on thrombophilia status in all participating patients, as in the study by Chaireti et al. ${ }^{11}$

Optimally, all patients would have been evaluated by diagnostic imaging on the day of sampling. However, since the study was performed within an existing clinical setting, the access to acute appointments for patients with discrete symptoms is somewhat limited and 20 patients were investigated one or more days later. However, we do not expect that this impacted outcomes. In our experience, venous thromboses will not be dissolved in a matter of days, so as to no longer be present in radiological exams. ${ }^{41}$ We also performed a 3-month follow-up of medical records, to decrease the risk of false negative diagnoses.

The use of platelet-free plasma is recommended for thrombin generation in hemophilia, ${ }^{29}$ because even traces of platelets can lead to overestimation of thrombin generation. This is especially troublesome in patients with low levels of thrombin generation, such as hemophilia patients. Since this study utilized samples collected for routine coagulation assays, these were platelet poor $\left(<10 \times 10^{9 / \mathrm{L}}\right)$, prepared by a stat-protocol with centrifugation at $3,000 \times g \times 10$ minutes. However, since all samples were prepared the same way, the study outcome of discrimination between clinical groups was not expected to be affected. In hypocoagulable patients, it is also recommended to use TF in low concentration $(\leq 1 \mathrm{M})$. In this study, we used the Innovance ETP B-setting, which are the proprietary recommended settings for patients with hypercoagulability.

\section{Conclusion}

In this cross-sectional diagnostic study, the OHP and Innovance ETP assays could not discriminate patients with VTE among emergency department outpatients with suspected VTE. The GHAs further indicated that the patients with suspected VTE were in a prothrombotic state, due in part to an increased fibrinogen level. These data suggest that OHP and ETP are sensitive to acute phase effects and comorbidities that are unavoidable in outpatients at the emergency department. In conclusion, the ETP and OHP do not seem to improve the diagnosis of acute VTE.

\section{Funding}

This study was supported by the Foundation for Coagulation Research at Karolinska Institutet, the Scandinavian Research Foundation for Varicose Veins and other Venous 
Diseases, FoU Region Stockholm, the Swedish Society on Thrombosis and Haemostasis with Leo Pharma.

\section{Conflict of Interest}

J.P.A. reports grants and other from CSL Behring, other from NovoNordisk, during the conduct of the study. All other authors have nothing to disclose.

\section{Acknowledgments}

The authors thank the medical staff from the Emergency Department at Karolinska University Hospital Huddinge and the laboratory staff at the coagulation laboratory.

\section{References}

1 Cohen AT, Gitt AK, Bauersachs R, et al. The management of acute venous thromboembolism in clinical practice. Results from the European PREFER in VTE Registry. Thromb Haemost 2017;117 (07):1326-1337

2 Smith-Bindman R, Lipson J, Marcus R, et al. Radiation dose associated with common computed tomography examinations and the associated lifetime attributable risk of cancer. Arch Intern Med 2009;169(22):2078-2086

3 Parfrey P. The clinical epidemiology of contrast-induced nephropathy. Cardiovasc Intervent Radiol 2005;28(Suppl 2):S3-S11

4 Refaai MA, Riley P, Mardovina T, Bell PD. The clinical significance of fibrin monomers. Thromb Haemost 2018;118(11):1856-1866

5 Farm M, Siddiqui AJ, Onelöv L, et al. Age-adjusted D-dimer cut-off leads to more efficient diagnosis of venous thromboembolism in the emergency department: a comparison of four assays. J Thromb Haemost 2018;16(05):866-875

6 Lippi G, Cervellin G, Casagranda I, Morelli B, Testa S, Tripodi A. Ddimer testing for suspected venous thromboembolism in the emergency department. Consensus document of AcEMC, CISMEL, SIBioC, and SIMeL. Clin Chem Lab Med 2014;52(05):621-628

7 Bratseth V, Pettersen Å, Opstad TB, Arnesen H, Seljeflot I. Markers of hypercoagulability in CAD patients. Effects of single aspirin and clopidogrel treatment. Thromb J 2012;10(01):12

8 Wexels F, Dahl OE, Pripp AH, Seljeflot I. Thrombin generation in patients with suspected venous thromboembolism. Clin Appl Thromb Hemost 2017;23(05):416-421

9 Haas FJ, Schutgens RE, Kluft C, Biesma DH. A thrombin generation assay may reduce the need for compression ultrasonography for the exclusion of deep venous thrombosis in the elderly. Scand J Clin Lab Invest 2011;71(01):12-18

10 Riva N, Vella K, Hickey K, et al. Biomarkers for the diagnosis of venous thromboembolism: D-dimer, thrombin generation, procoagulant phospholipid and soluble P-selectin. J Clin Pathol 2018; 71(11):1015-1022

11 Chaireti R, Jennersjö C, Lindahl TL. Thrombin generation and Ddimer concentrations in a patient cohort investigated for venous thromboembolism. Relations to venous thrombosis, factor V Leiden and prothrombin G20210A. The LIST study. Thromb Res 2009; 124(02):178-184

12 Hunt BJ, Parmar K, Horspool K, Shephard N, Nelson-Piercy C, Goodacre S; DiPEP research group. The DiPEP (Diagnosis of PE in Pregnancy) biomarker study: an observational cohort study augmented with additional cases to determine the diagnostic utility of biomarkers for suspected venous thromboembolism during pregnancy and puerperium. Br J Haematol 2018;180 (05):694-704

13 Segers O, van Oerle Rv, ten Cate Ht, Rosing J, Castoldi E. Thrombin generation as an intermediate phenotype for venous thrombosis. Thromb Haemost 2010;103(01):114-122

14 Lutsey PL, Folsom AR, Heckbert SR, Cushman M. Peak thrombin generation and subsequent venous thromboembolism: the Lon- gitudinal Investigation of Thromboembolism Etiology (LITE) study. J Thromb Haemost 2009;7(10):1639-1648

15 Eichinger S, Heinze G, Jandeck LM, Kyrle PA. Risk assessment of recurrence in patients with unprovoked deep vein thrombosis or pulmonary embolism: the Vienna prediction model. Circulation 2010;121(14):1630-1636

16 van Hylckama Vlieg A, Baglin CA, Luddington R, MacDonald S, Rosendaal FR, Baglin TP. The risk of a first and a recurrent venous thrombosis associated with an elevated D-dimer level and an elevated thrombin potential: results of the THE-VTE study.J Thromb Haemost 2015;13(09):1642-1652

17 Pepperell D, Morel-Kopp M-C, Ward C. Clinical application of fibrinolytic assays. In: Kolev K, ed. Fibrinolysis and Thrombolysis. Rijeka, Croatia: Intech; 2014:139-148

18 Antovic JP, Mikovic D, Elezovic I, et al. Two global haemostatic assays as additional tools to monitor treatment in cases of haemophilia A. Thromb Haemost 2012;108(01):21-31

19 Antovic A. Screening haemostasis-looking for global assays: the Overall Haemostasis Potential (OHP) method-a possible tool for laboratory investigation of global haemostasis in both hypo- and hypercoagulable conditions. Curr Vasc Pharmacol 2008;6(03):173-185

20 Vikerfors A, Svenungsson E, Ågren A, et al. Studies of fibrin formation and fibrinolytic function in patients with the antiphospholipid syndrome. Thromb Res 2014;133(05):936-944

21 Chow V, Reddel C, Pennings G, et al. Persistent global hypercoagulability in long-term survivors of acute pulmonary embolism. Blood Coagul Fibrinolysis 2015;26(05):537-544

22 Curnow JL, Morel-Kopp MC, Roddie C, Aboud M, Ward CM. Reduced fibrinolysis and increased fibrin generation can be detected in hypercoagulable patients using the overall hemostatic potential assay. J Thromb Haemost 2007;5(03):528-534

23 Edelman JJ, Reddel CJ, Kritharides L, et al. Natural history of hypercoagulability in patients undergoing coronary revascularization and effect of preoperative myocardial infarction. J Thorac Cardiovasc Surg 2014;148(02):536-543

24 Rooth E, Wallén H, Antovic A, et al. Thrombin activatable fibrinolysis inhibitor and its relationship to fibrinolysis and inflammation during the acute and convalescent phase of ischemic stroke. Blood Coagul Fibrinolysis 2007;18(04):365-370

25 Lim HY, O'Malley C, Donnan G, Nandurkar H, Ho P. A review of global coagulation assays - is there a role in thrombosis risk prediction? Thromb Res 2019;179:45-55

26 Bombardier C, Villalobos-Menuey E, Ruegg K, Hathaway WE, Manco-Johnson MJ, Goldenberg NA. Monitoring hypercoagulability and hypofibrinolysis following acute venous thromboembolism in children: application of the CloFAL assay in a prospective inception cohort study. Thromb Res 2012;130(03):343-349

27 Siemens Healtcare Diagnostics, Innovance ETP. Kit Insert, 2018. OPGAG03C0104(1113)

$28 \mathrm{He} \mathrm{S}$, Bremme K, Blombäck M. A laboratory method for determination of overall haemostatic potential in plasma. I. Method design and preliminary results. Thromb Res 1999;96(02):145-156

29 Dargaud Y, Wolberg AS, Gray E, Negrier C, Hemker HC; Subcommittee on Factor VIII, Factor IX, and Rare Coagulation Disorders. Proposal for standardized preanalytical and analytical conditions for measuring thrombin generation in hemophilia: communication from the SSC of the ISTH. J Thromb Haemost 2017;15(08): 1704-1707

30 Devreese K, Wijns W, Combes I, Van kerckhoven S, Hoylaerts MF. Thrombin generation in plasma of healthy adults and children: chromogenic versus fluorogenic thrombogram analysis. Thromb Haemost 2007;98(03):600-613

31 He S, Antovic A, Blombäck M. A simple and rapid laboratory method for determination of haemostasis potential in plasma. II. Modifications for use in routine laboratories and research work. Thromb Res 2001;103(05):355-361

32 Roche Diagnostics, Tina-quant D-Dimer Gen.2. 2015-10. 0004912551190 COINV50 
e188 Diagnostic Accuracy of DDI, ETP, OHP, and FM Farm et al.

33 Westerlund E, Woodhams BJ, Eintrei J, Söderblom L, Antovic JP. The evaluation of two automated soluble fibrin assays for use in the routine hospital laboratory. Int J Lab Hematol 2013;35(06):666-671

34 Medirox. MRX Owren's PT. Revision: 2018-08-20

35 Clauss A. Gerinnungsphysiologische schnellmetode zur bestimmung des fibrinogens. Acta Haematol 1957;17:237-246

36 Siemens Healthcare Diagnostics, Berichrom ${ }^{\circledR}$ Antitrombin III (A). 2013-07 OWWRG17E33 Rev. 03

37 Zweig MH, Campbell G. Receiver-operating characteristic (ROC) plots: a fundamental evaluation tool in clinical medicine. Clin Chem 1993;39(04):561-577

38 Clinical and Laboratory Standards Institute (CLSI), Quantitative Ddimer for exclusion of venous thromboembolic disease; approved guideline. CLSI document H59-A. Clinical and Laboratory Standards Institute 950 West Valley Road, Suite 2500 Wayne, PA 19087 USA; $2011 ; 31(6)$

39 Kintigh J, Monagle P, Ignjatovic V. A review of commercially available thrombin generation assays. Res Pract Thromb Haemost 2017;2(01):42-48

40 Fleck D, Albadawi H, Wallace A, Knuttinen G, Naidu S, Oklu R. Below-knee deep vein thrombosis (DVT): diagnostic and treatment patterns. Cardiovasc Diagn Ther 2017;7(Suppl 3): S134-S139

41 Holmström M, Lindmarker P, Granqvist S, Johnsson H, Lockner D. A 6-month venographic follow-up in 164 patients with acute deep vein thrombosis. Thromb Haemost 1997;78(02):803-807 